\title{
Hsp90 inhibition increases p53 expression and destabilizes MYCN and MYC in neuroblastoma
}

\author{
PAUL L. REGAN, JOSHUA JACOBS, GERALD WANG, JAIME TORRES, \\ ROBBY EDO, JENNIFER FRIEDMANN and XAO X. TANG \\ Department of Anatomy and Cell Biology, College of Medicine, University of Illinois at Chicago, Chicago, IL, USA
}

Received July 29, 2010; Accepted September 6, 2010

DOI: 10.3892/ijo_00000829

\begin{abstract}
Neuroblastoma is a childhood cancer that exhibits either a favorable or an unfavorable phenotype. MYCN and MYC are oncoproteins that play crucial roles in determining the malignancy of unfavorable neuroblastoma. The Hsp90 superchaperone complex assists in the folding and function of a variety of oncogenic client proteins. Inhibition of Hsp90 by small molecule inhibitors leads to the destabilization of these oncogenic proteins and consequently suppresses tumor malignancy. Nonetheless, little is known about the effect of Hsp90 inhibition on the stability of MYCN and MYC proteins. In this study, we investigated the effect of Hsp90 inhibition on the phenotype of unfavorable neuroblastoma cells including its effect on MYCN and MYC expression. Two $M Y C N$-amplified neuroblastoma cell lines (IMR5 and CHP134) and two non-MYCN-amplified cell lines (SY5Y and SKNAS) were used to address the effect of Hsp90 inhibition on the malignant phenotype of neuroblastoma. It was found that Hsp90 inhibition in neuroblastoma cell lines resulted in significant growth suppression, a decrease in MYCN and MYC expression, and an increase in the expression of p53. In the TP53-mutated SKNAS cell line, Hsp90 inhibition enhanced the expression of the favorable neuroblastoma genes EFNB2, MIZ-1 and NTRK1 (TrkA). In addition, Hsp90 inhibition reduced HDAC6 expression and enhanced tubulin acetylation. Together our data suggest that Hsp90 inhibition suppresses the growth of neuroblastoma through multiple cellular pathways and that MYC/MYCN destabilization is among the important consequences of Hsp90 inhibition.
\end{abstract}

Correspondence to: Dr Xao X. Tang, Department of Anatomy and Cell Biology, 808 S. Wood Street, Room 578 CME (MC 512), Chicago, IL 60612, USA

E-mail: xaotang@uic.edu

Abbreviations: 17-DMAG, 17-dimethylaminoethylamino-17demethoxy-geldanamycin

Key words: Hsp90 inhibition, neuroblastoma, MYCN, MYC destabilization

\section{Introduction}

Neuroblastoma is a neural crest-derived tumor and is the most common extracranial pediatric malignancy. The tumor accounts for $7-10 \%$ of all childhood cancers and is the cause of $\sim 15 \%$ of fatalities in children with cancer. Neuroblastoma is unique because of its propensity to exhibit either a favorable or an unfavorable phenotype. Favorable neuroblastomas can undergo spontaneous regression or maturation. These tumors are also curable by surgical removal with or without adjuvant chemotherapy. In contrast, unfavorable neuroblastomas exhibit unrestrained growth despite the most intensive treatment $(1,2)$.

About half of unfavorable neuroblastomas are $M Y C N$ amplified and express high levels of MYCN. MYCN amplification is associated with rapid tumor progression and the worst disease outcome (3-5). A recent report suggests that in non- $M Y C N$-amplified unfavorable neuroblastomas, MYC rather than MYCN expression provides the aggressive phenotype (6). There is also a clear-cut dichotomy that $M Y C N$ amplified neuroblastoma cell lines express MYCN, whereas non-MYCN-amplified neuroblastoma cell lines express MYC at high levels. These observations suggest that MYCN or MYC expression is one of the major determining factors of neuroblastoma malignancy.

The concept of favorable neuroblastoma genes was first introduced in our previous study (7). High-level expression of favorable neuroblastoma genes is associated with good neuroblastoma disease outcome. In addition, forced expression of these genes in unfavorable neuroblastoma cells results in growth suppression. Notably, $M Y C N$-amplified neuroblastomas, the most aggressive form of the tumor, exhibit little or no expression of these genes. Thus far, several favorable neuroblastoma genes have been identified, which include EPHB6, EFNB2, EFNB3, NTRK1 (TrkA), CD44 and MIZ-1 (7-12). We have previously reported that known favorable neuroblastoma genes are epigenetically silenced in unfavorable neuroblastoma cells (13). In addition, our study suggests that favorable neuroblastoma gene expressions can be considered molecular indicators of the effectiveness of chemotherapeutic agents against neuroblastoma cells (9).

Hsp90 is essential for maintaining the conformational maturation, stability and activity of client proteins, including many key proteins necessary for the oncogenic phenotype. These proteins include BCR-ABL, ERBB2, EGFR, CRAF, 
BRAF, AKT, MET, VEGFR, FLT3, androgen and estrogen receptors, HIF-1 $\alpha$, and telomerase. Inhibition of Hsp90 by small-molecule inhibitors leads to destabilization of its client oncogenic proteins and consequently suppresses tumor malignancy (14). Nonetheless, there has been little information on the effect of Hsp90 inhibition on the stability of MYC and MYCN proteins.

Studies on the effect of Hsp90 inhibition in neuroblastoma have also been limited. It was reported that an Hsp90 inhibitor, geldanamycin, depleted AKT and IGF1R and suppressed growth of non-MYCN-amplified SK-N-SH and $M Y C N$-amplified IMR32 human neuroblastoma cell lines in vitro (15). The effect of Hsp90 inhibition in preclinical test settings has generated mixed results so far. It was shown that Hsp90 inhibitors 17-AAG and EC5 had growth suppressive effects on xenografts of two neuroblastoma cell lines, SK-NSH and LAN-1 (MYCN-amplified) (16). In contrast, a limited efficacy of 17-DMAG on xenografts of several neuroblastoma cell lines was later reported (17). None of these studies examined the expression of MYC and MYCN proteins as indicators of the malignancy of neuroblastoma cells in culture or xenografts in response to Hsp90 inhibition. In this study, we have shown that Hsp90 inhibition suppresses the malignant phenotype of unfavorable neuroblastoma cells by downregulating MYCN and MYC, increasing p53 expression, and enhancing tubulin acetylation as well as the expression of favorable neuroblastoma genes.

\section{Materials and methods}

Neuroblastoma cell lines. The neuroblastoma cell lines (IMR5, CHP134, SY5Y and SKNAS) were grown in RPMI-1640 supplemented with $5 \%$ fetal bovine serum and OPI (1 mM oxaloacetate, $0.45 \mathrm{mM}$ pyruvate, $0.2 \mathrm{U} / \mathrm{ml}$ insulin, final concentration). These cell lines tested negative for mycoplasma, and their identity was validated by the original source. IMR5 (a clone of IMR32) and CHP134 were received from Dr Roger H. Kennett (Wheaton College, Wheaton, IL). SY5Y was the gift from Dr Robert Ross (Fordham University, Bronx, NY). SKNAS was from Dr C. Patrick Reynolds (The Texas Tech University Health Sciences Center, Lubbock, TX).

MTS assay. An MTS [3-(4,5-dimethylthiazol-2-yl)-5-(3-carboxymethoxyphenyl)-2-(4-sulfophenyl)-2H-tetrazolium, inner salt] assay (a water soluble form of the MTT assay) was performed as described in our previous study (7). 17-(dimethylaminoethylamino)-17-demethoxygeldanamycin hydrochloride (17-DMAG) was purchased from LC Laboratories, Woburn, MA, USA. The stock solution was made at $2.5 \mathrm{mM}$ in $\mathrm{H}_{2} \mathrm{O}$, filter-sterilized and stored at $-20^{\circ} \mathrm{C}$.

Western blot analysis. Western blotting was performed according to the method previously described (18) except SuperSignal West Dura extended duration substrate (Pierce) was used. Light emission signals were captured by an LAS3000 (Fujifilm) digital image analyzer. Cell extracts were made in 2-D gel sample buffer (9 M urea, 2\% Nonidet-P40,

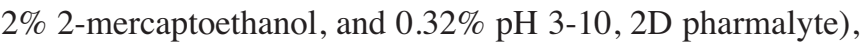
and the protein content of the samples was determined by the
BioRad protein assay kit using bovine serum albumin as a standard and the sample buffer as the blank. Antibodies used to detect proteins of interest are described in the figure legends.

Reverse transcription and TaqMan real-time PCR. RNAs were isolated from neuroblastoma cell lines using the Qiagen RNeasy kit. Total RNA ( $2 \mu \mathrm{g}$ ) was used to synthesize cDNA. The experimental procedures for the reverse transcription were performed as previously described (19). The quantitative real-time PCR was done using an iQ5 real-time PCR machine (BioRad). TaqMan probes were purchased from Applied Biosystems, Inc., and the multiplex qPCR mix (QuantiTect Multiplex PCR kit) was purchased from Qiagen. Relative quantification of expression levels of genes of interest was done by the $\Delta \Delta \mathrm{Ct}$ method using the expression of GAPD RNA as an internal control. The experimental procedures were performed according to the instructions provided by Qiagen and BioRad.

Subcellular fractionation. Cell pellets washed in Dulbecco's modified phosphate-buffered saline (D-PBS) were resuspended in D-PBS containing $0.5 \%$ Nonidet P-40 ( $\mathrm{vol} / \mathrm{vol}$ ) and $1 \%(\mathrm{v} / \mathrm{v})$ Sigma proteinase inhibitor cocktail (P8340) by pipetting 20 times using a $200 \mu 1$ Rainin pipetter. The resulting homogenates were centrifuged for $60 \mathrm{sec}$ in an Eppendorf microfuge at $100 \mathrm{rcf}$. The supernatants contain the cytoplasm, membrane and mitochondria fractions, and the pellets contain the nuclear fraction. The pellets were further washed in the above solution and centrifuged in the same fashion. The supernatant was collected and designated as the nuclear wash fraction. The resultant pellets were extracted with the 2-D gel sample buffer (see Western blot section), and the cleared supernatants, after being centrifuged at 13,200 rpm for $5 \mathrm{~min}$ in an Eppendorf centrifuge were designated as the nuclear fraction.

Transient transfection of neuroblastoma cells with MIZ-1. Full-length cDNA of MIZ- 1 was cloned into an eukaryotic expression vector, pEAK12. The neuroblastoma cells indicated (Fig. 8) were transfected with the pEAK/MIZ-1 construct by electroporation using an XCell electroporator (BioRad) (120 V square wave for $20 \mathrm{msec}$ ). To examine MIZ-1 protein expression by Western blot analysis and 2-D gel analysis, the cells were harvested at $24 \mathrm{~h}$ after transfection.

2-D gel analysis. The 2-D gel electrophoresis was done according to the ReadyPrep ${ }^{\mathrm{TM}}$ 2-D Starter Kit and PROTEAN ${ }^{\circledR}$ IEF cell instruction manuals. Briefly, cell extracts for 2-D gel electrophoresis were made in the 2-D sample buffer (see Western blot section). An 11-cm, pH 3.0-10, immobilized pH gradient (IPG) strip was re-hydrated directly with $200 \mu 1$ ReadyPrep rehydration/sample buffer, which included $50 \mu \mathrm{g}$ cell extract at room temperature, overnight. The re-hydrated IPG strips were then placed on a PROTEAN IEF cell and the first dimension electrophoresis was performed using the rapid voltage ramping program. After the first dimension electrophoresis, the IPG strips were equilibrated consecutively with Equilibration Buffer I (BioRad) and with Equilibration Buffer II containing iodoacetamide (BioRad). The IPG strips were then placed on $4-20 \%$ Criterion pre-cast gels and the 


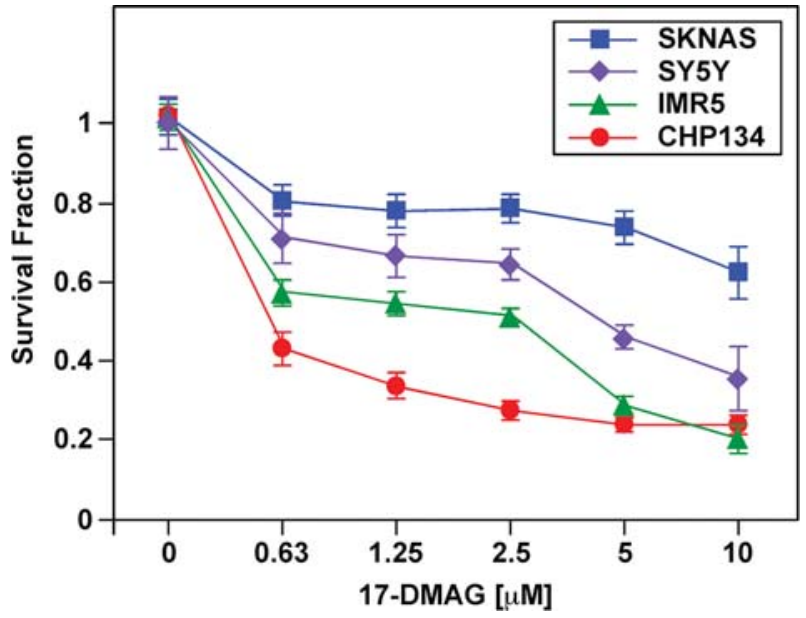

Figure 1. Inhibition of Hsp90 by 17-DMAG results in significant growth suppression of neuroblastoma cell lines. Neuroblastoma cells were treated with 17-DMAG at the concentrations indicated. Two days after the treatments, an MTS assay was done to determine the effect of the drug on growth of the neuroblastoma cell lines indicated.

second dimension electrophoresis was performed using a Criterion Cell (BioRad).

\section{Results}

Hsp90 inhibition results in growth suppression of unfavorable neuroblastoma cells. All neuroblastoma cell lines to date are derived from unfavorable neuroblastomas. To examine the effect of Hsp90 inhibition on growth of unfavorable neuroblastoma cells, the four cell lines IMR5, CHP134, SY5Y and SKNAS were used. IMR5 and CHP134 are $M Y C N$-amplified neuroblastoma cell lines and express high levels of MYCN. SY5Y and SKNAS are non- $M Y C N$-amplified cell lines and express high levels of MYC. 17-DMAG was used as a model agent for Hsp90 inhibitors because of its water solubility and potency. As shown in Fig. 1, 17-DMAG inhibited growth of the four neuroblastoma cell lines in dose-dependent fashions after two days of the treatment. Among the cell lines, CHP134 was most sensitive to 17-DMAG treatments, whereas SKNAS (a TP53-mutated line) was least sensitive to the treatments. In addition, there was a biphasic growth inhibitory effect of Hsp90 inhibition for SKNAS, SY5Y and IMR5. In these three cell lines, 17-DMAG showed similar growth inhibitory effects between the concentrations of 0.63 and $2.5 \mu \mathrm{M}$, and its effect was further enhanced up to $10 \mu \mathrm{M}$ according to the dose. Based on these results, subsequent assays were done using 17-DMAG at the dose of $5 \mu \mathrm{M}$ for all neuroblastoma cell lines.

The effect of Hsp90 inhibition on MYCN and MYC destabilization in neuroblastoma cell lines. It has been shown that inhibition of Hsp90 leads to the down-regulation of known oncoproteins, including AKT, ERBB2, BRAF and BCRABL (14). Nonetheless, whether or not Hsp90 inhibition can affect MYC and MYCN stability has not been well documented. In this study, we examined whether the growth suppressive effect of Hsp90 inhibition on the neuroblastoma cells was associated with MYCN and MYC destabilization in
A
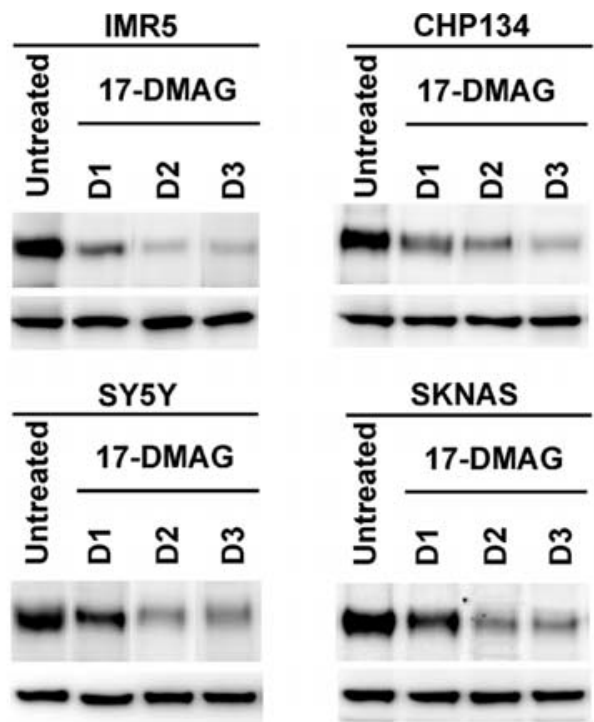

MYCN

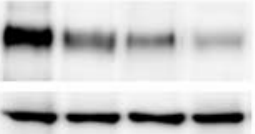

$\beta$-Actin

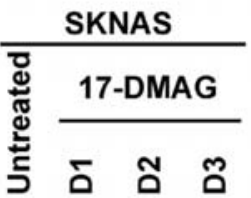

MYC

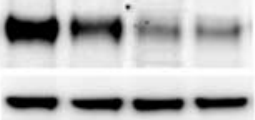

$\beta$-Actin
B
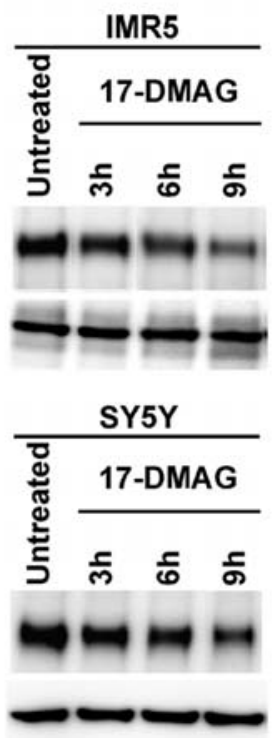

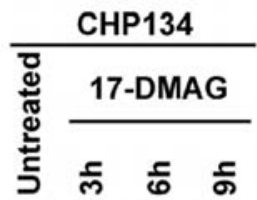

MYCN

$\beta$-Actin

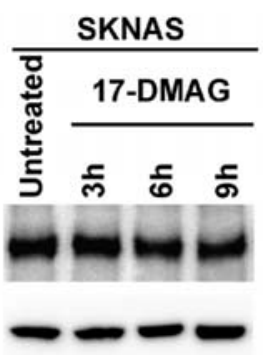

MYC

$\beta$-Actin
Figure 2. Treatment of neuroblastoma cells with 17-DMAG results in a decrease in MYCN expression (IMR5 and CHP134) and MYC expression (SY5Y and SKNAS). (A) IMR5, CHP134, SY5Y and SKNAS were treated with 17-DMAG $(5 \mu \mathrm{M})$ for 1,2 and 3 days. The cells were harvested and subjected to Western blot analysis. Total protein $(5 \mu \mathrm{g})$ was loaded per lane. The MYCN-specific monoclonal antibody, NCM II 100, was used to detect expression of MYCN (38). The MYC-specific monoclonal antibody (9E10, ATCC) and anti- $\beta$-actin monoclonal antibody (C4, Santa Cruz Biotechnology) were used to detect expression of MYC and B-actin, respectively. (B) Time course studies were performed to determine the expression of MYCN or MYC in the neuroblastoma cells after 3, 6 and $9 \mathrm{~h}$ of 17-DMAG treatment as described in $(\mathrm{A})$

these cells. As shown in Fig. 2A, treatment of these cell lines with 17-DMAG resulted in a clear decrease in MYCN or MYC expression as early as day 1 of the treatment. Early time course studies showed that the effect of the drug treatment on MYCN and MYC stability varied among the cell lines examined (Fig. 2B). The drug treatment was most effective against MYCN and MYC in IMR5 and SY5Y, respectively. MYCN and MYC down-regulation was clearly observed in IMR5 and SY5Y as early as $3 \mathrm{~h}$ of the drug treatment. A small 
A

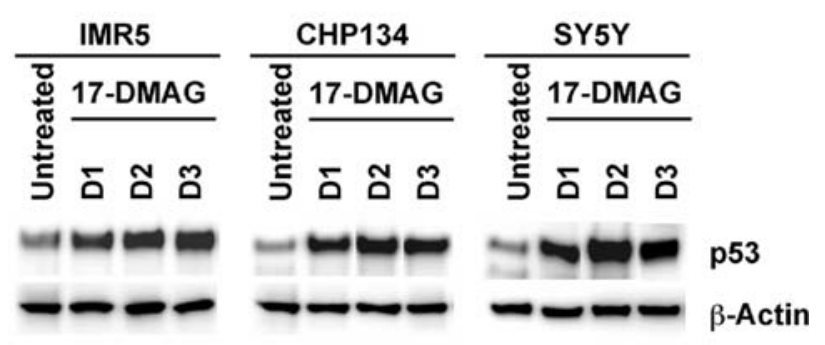

B

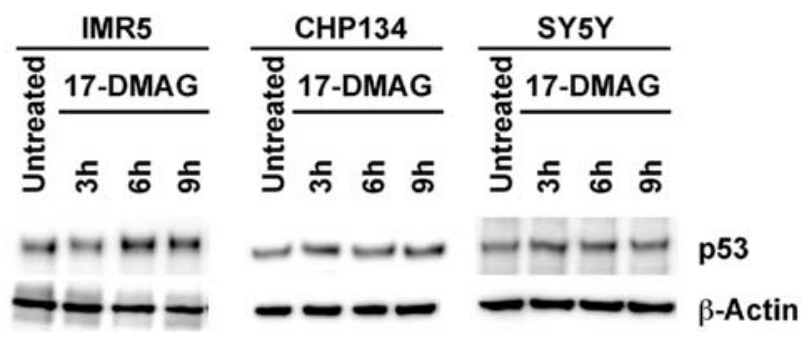

Figure 3. Treatment of neuroblastoma cells with 17-DMAG results in an increase in p53 expression in IMR5, CHP134 and SY5Y. (A) IMR5, CHP134 and SY5Y were treated with 17-DMAG $(5 \mu \mathrm{M})$ for 1,2 and 3 days. The cells were harvested and subjected to Western blot analysis. Total protein $(5 \mu \mathrm{g})$ was loaded per lane. A p53-specific monoclonal antibody, DO-1 (Calbiochem), was used to detect expression of p53. (B) Time-course studies were performed to determine the expression of p53 in the neuroblastoma cells after 3,6 and $9 \mathrm{~h}$ of 17-DMAG treatment as described in (A).

reduction of MYCN and MYC expression was also seen in CHP134 and SKNAS treated with 17-DMAG for 3 and $9 \mathrm{~h}$, respectively.

Inhibition of $H$ sp 90 results in an increased p53 expression in neuroblastoma cell lines. Our previous study indicated that an elevated p53 expression had a suppressive effect on MYCN expression in $M Y C N$-amplified neuroblastoma cells (20). We thus examined if Hsp90 inhibition by 17-DMAG could upregulate p53 expression in neuroblastoma cell lines. The SKNAS cell line was not included in this experiment because it harbors TP53 mutations $(21,22)$. As shown in Fig. 3A, treatment of IMR5, CHP134 and SY5Y with 17-DMAG in fact resulted in an increased p53 expression as early as day 1 of the treatment. Early time course studies showed that the effect of the drug treatments on p53 expression varied among the cell lines examined. An enhancement of p53 expression was most apparent in IMR5, in which p53 expression was increased after $6 \mathrm{~h}$ of the drug treatment (Fig. 3B). There was no apparent effect on p53 expression in CHP134 and SY5Y up to $9 \mathrm{~h}$ of the drug treatment.

The effect of Hsp90 inhibition on expression of $p 21^{\text {WAFI }}$ in neuroblastoma cell lines. As described, Hsp90 inhibition increased p53 expression in the neuroblastoma cells (Fig. 3). We therefore examined if 17-DMAG treatment up-regulated the expression of $\mathrm{p} 21^{\mathrm{WAF} 1}$, a known target of $\mathrm{p} 53$. As shown in Fig. 4, Hsp90 inhibition by 17-DMAG resulted in an upregulation of $\mathrm{p} 21^{\mathrm{WAF} 1}$ expression in IMR5 and SY5Y cells, but not in CHP134. SKNAS with TP53 mutations showed little induction of $\mathrm{p} 21^{\mathrm{WAF} 1}$ expression upon the drug treatment (see Discussion).

The effect of Hsp90 inhibition on AKT expression in neuroblastoma cell lines. AKT is a known client protein of Hsp90, and thus inhibition of Hsp90 leads to degradation of AKT (23-25). In addition, the AKT pathway is known to stabilize MYC (26) and MYCN (27). We thus examined the effect of Hsp90 inhibition by 17-DMAG on AKT stability in the neuroblastoma cells as a control, and to compare to the MYCN and MYC destabilization described in Fig. 2A. As shown in Fig. 5A, 17-DMAG treatment of the neuroblastoma cells resulted in a decreased AKT expression. Kinetics of AKT destabilization resembled to those of MYCN and MYC down-regulation in the neuroblastoma cell lines examined (Fig. 2A). In addition, Hsp90 inhibition by 17-DMAG treatments did not change the subcellular localization of AKT, MYCN and MYC in CHP134 and SKNAS cells (Fig. 5B). Subcellular localization of these proteins in the drug-treated IMR5 and SY5Y was not examined.

17-DMAG enhances tubulin acetylation in neuroblastoma cells and such effect is accompanied by a reduction of HDAC6. To address a potential role of Hsp90 inhibition in interfering with mitosis, we examined the expression of acetylated tubulin in the 17-DMAG-treated neuroblastoma cells. As shown in Fig. 6, there was an increased expression of acetylated tubulin in the drug-treated cells, suggesting that tubulin deacetylase levels were down-regulated by Hsp90 inhibition. In fact, expression levels of a tubulin deacetylase, HDAC6, were markedly suppressed in these cells (Fig. 6).

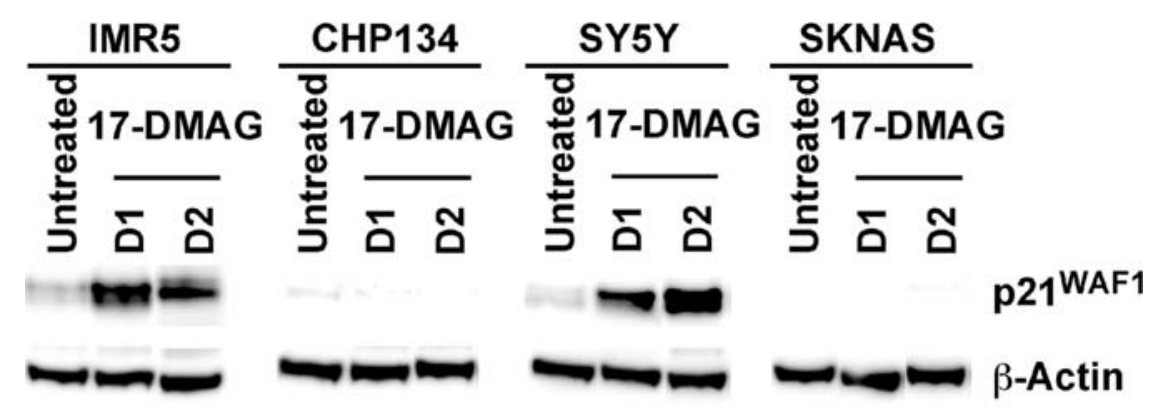

Figure 4 . The effect of 17-DMAG on $\mathrm{p} 21^{\mathrm{WAF} 1}$ expression in neuroblastoma cells. IMR5, CHP134, SY5Y and SKNAS were treated with $17-\mathrm{DMAG}$ (5 $\mu$ M) for 1 and 2 days. The cells were harvested and subjected to Western blot analysis. For p21 WAF1 expression, total protein (20 $\mu \mathrm{g})$ was loaded per lane. For $\beta$-actin expression, total protein $(5 \mu \mathrm{g})$ was loaded per lane. A mouse monoclonal antibody (EA10, Calbiochem) was used to detect expression of p21 ${ }^{\text {WAF1 }}$ protein. 
A

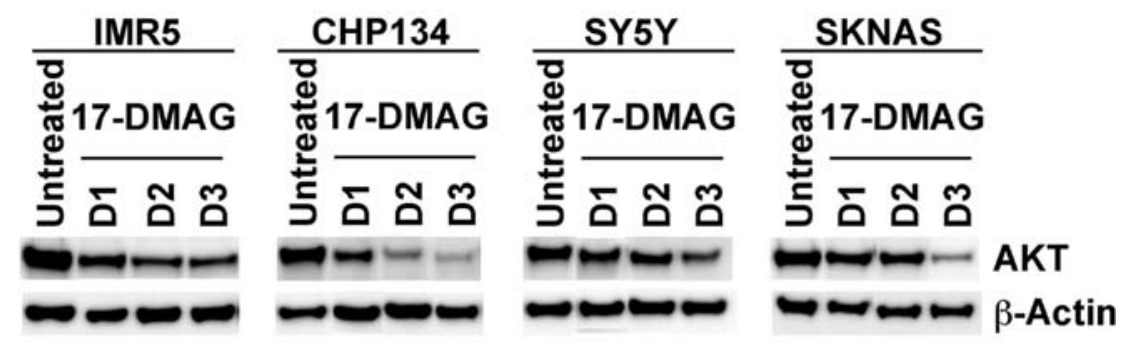

B

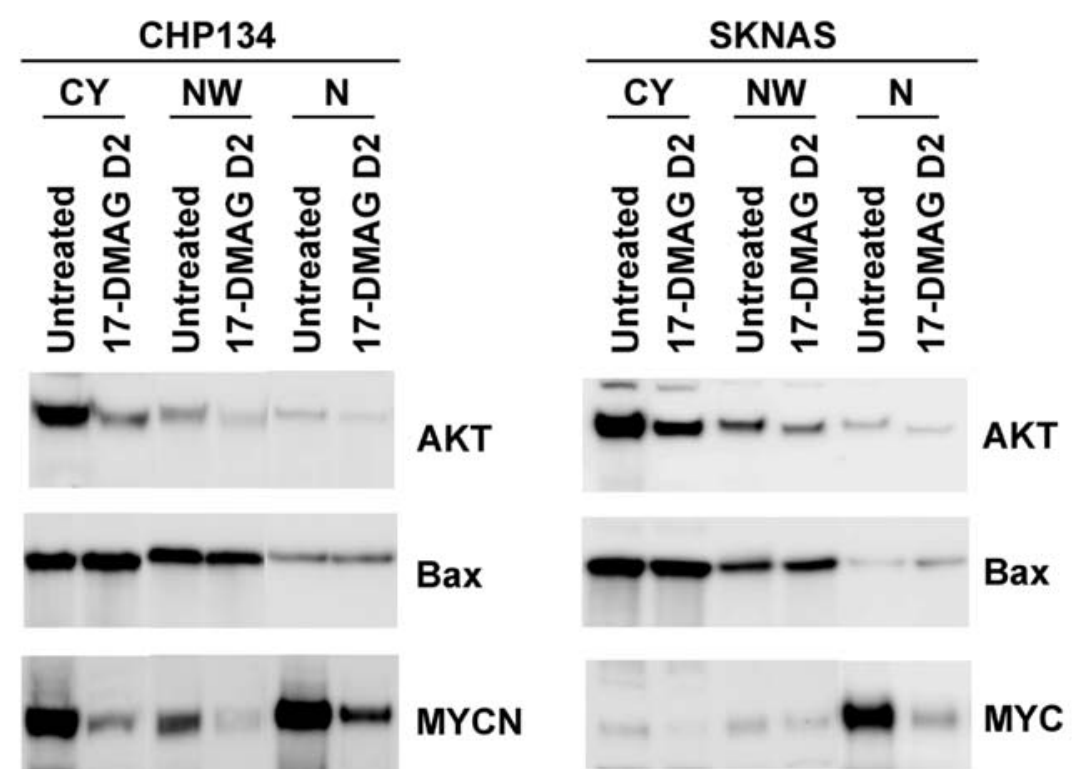

Figure 5. (A) The effect of 17-DMAG on AKT expression in neuroblastoma cells. IMR5, CHP134, SY5Y and SKNAS were treated with 17-DMAG (5 $\mu$ M) for 1,2 and 3 days. The cells were harvested and subjected to Western blot analysis. Total protein $(5 \mu \mathrm{g})$ was loaded per lane. An anti-AKT rabbit polyclonal antibody (Cell Signaling) was used to detect the expression of AKT protein. (B) Subcellular localization of AKT in the 17-DMAG-treated CHP134 and SKNAS cells. The expressions of Bax, MYCN and MYC were included as controls for the mitochondria/cytoplasm fraction and nucleus fraction, respectively. An equal proportion of each fraction [cytoplasm (CY), nucleus wash (NW) and nucleus $(\mathrm{N})$ ] was analyzed by Western blot analysis using the anti-AKT rabbit polyclonal antibody as described in (A), the anti-MYCN NCM II 100 antibody, the anti-MYC 9E10 antibody, and the anti-Bax rabbit polyclonal antibody (N-20, Santa Cruz Biotechnology).

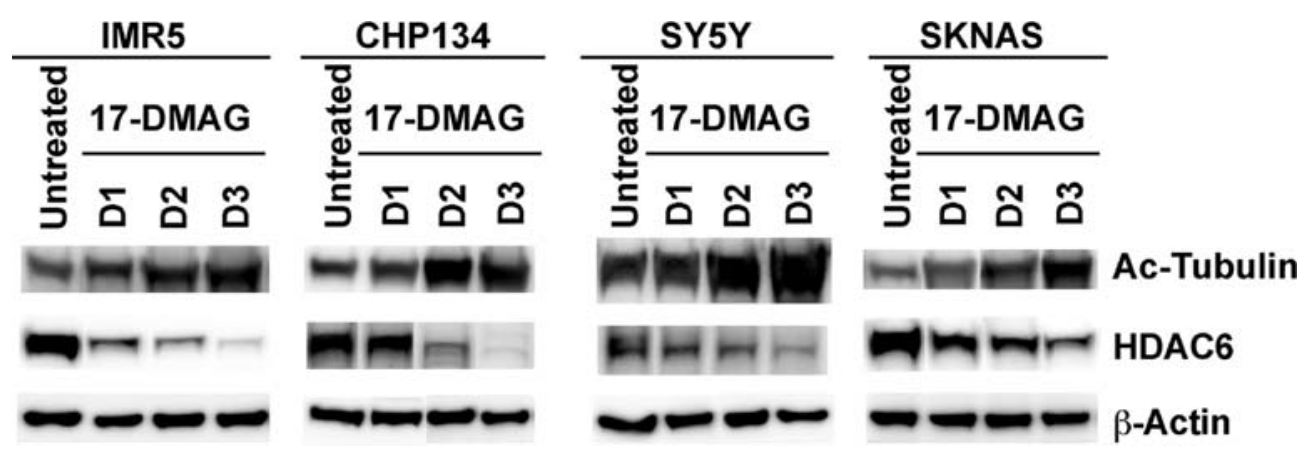

Figure 6. Treatment of neuroblastoma cell lines with 17-DMAG results in an enhancement of tubulin acetylation and a reduction of HDAC6 expression. IMR5, CHP134, SY5Y and SKNAS were treated with 17-DMAG (5 $\mu \mathrm{M})$ for 1, 2 and 3 days. The cells were harvested and subjected to Western blot analysis. For acetylated tubulin expression, total protein $(15 \mu \mathrm{g})$ was loaded per lane. For HDAC6 expression, total protein $(20 \mu \mathrm{g})$ was loaded per lane. For $\beta$-actin expression, total protein $(5 \mu \mathrm{g})$ was loaded per lane. An acetylated tubulin-specific monoclonal antibody (6-11B-1, Sigma) and an HDAC6-specific mouse monoclonal antibody (D-11, Santa Cruz Biotechnology) were used to detect expression of proteins of interest.

Treatment of SKNAS cells with 17-DMAG results in an increased expression of favorable neuroblastoma genes EFNB2, MIZ-1, NTRK1 (TrkA) and growth suppressive genes $N R G 1$, SELIL. Favorable neuroblastoma genes are known to be growth suppressive $(7,9,28,29)$. Since SKNAS is a TP53mutated cell line, we asked whether Hsp90 inhibition upregulated favorable neuroblastoma genes in SKNAS as an alternative mechanism to $\mathrm{p} 53$ pathways in suppressing growth 


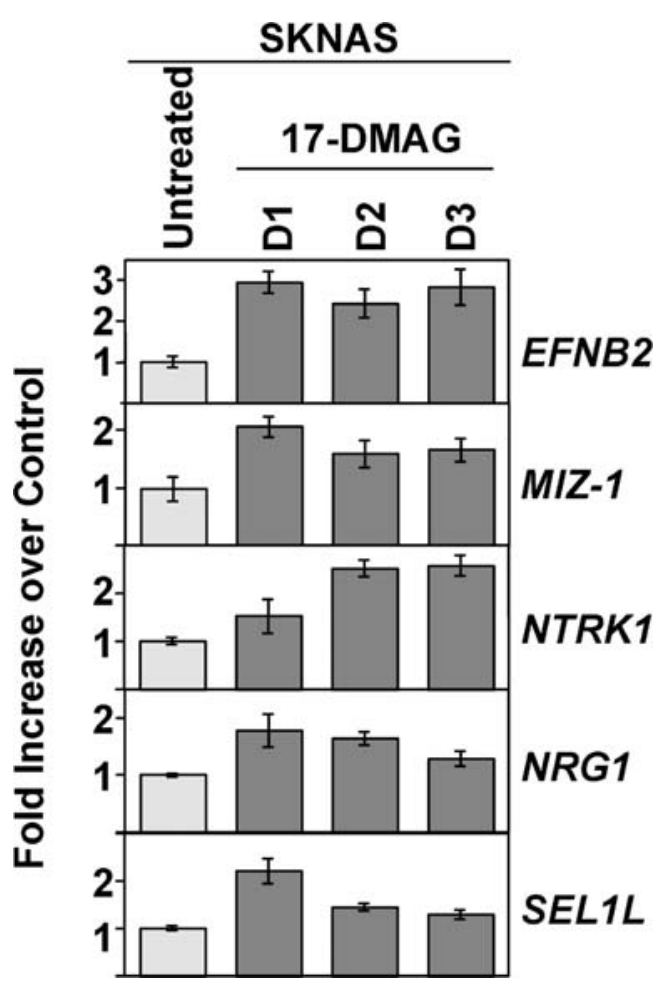

Figure 7. Enhanced expression of favorable neuroblastoma genes (EFNB2, $M I Z-1, N T R K 1)$ and growth suppressive genes $(N R G 1, S E L 1 L)$ in 17-DMAGtreated SKNAS. SKNAS cells were treated with 17-DMAG $(5 \mu \mathrm{M})$ for 1,2 and 3 days. RNAs were prepared from the control untreated cells and the drug-treated cells. Expression of genes of interest was examined in duplicate by TaqMan real-time PCR using gene-specific TaqMan Gene Expression Assays (ABI). Expression levels of EFNB2, MIZ-1, NTRK1, NRG1, SEL1L were presented as fold increase in the 17-DMAG-treated SKNAS cells over the untreated control

of these cells. As shown in Fig. 7, treatment of SKNAS cells with 17-DMAG resulted in an increased expression of favorable neuroblastoma genes $(E F N B 2, M I Z-1, N T R K 1)$ as well as growth suppressive genes $(N R G 1, S E L 1 L)(30,31)$.

The effect of Hsp90 inhibition on MIZ-1 protein expression. Thus far, MIZ- 1 is the only known favorable neuroblastoma gene to encode a transcription factor (9). Previous studies from our group and others suggest that MIZ-1 positively regulates expression of other favorable neuroblastoma genes (9) and genes encoding CDK inhibitors (CDKN1A, CDKN2B) (32-34). We thus investigated if MIZ-1 protein expression was also up-regulated in the 17-DMAG-treated cell lines. As shown in Fig. 8, MIZ-1 protein was detected in the four cell lines treated with 17-DMAG. However, it was noted that treatment of these cells with 17-DMAG induced a smaller molecular weight MIZ-1 protein ( $90 \mathrm{kDa})$ as compared to that of MIZ-1 detected in MIZ-1-transfected cells ( 125-130 kDa). In addition, results shown in Fig. 8 were reproducible when different anti-MIZ-1 antibodies were used (Fig. 8 legend). It should be noted that based on the deduced amino acid sequence of MIZ-1, its expected molecular weight is $88 \mathrm{kDa}$. To further confirm data shown in Fig. 8, we performed 2-D gel analysis using CHP134 and SKNAS treated with 17-DMAG. As shown in Fig. 9, 17-DMAG did in fact induce MIZ-1 protein in these cell lines, but the drug-induced MIZ-1 protein had a smaller molecular weight ( $90 \mathrm{kDa})$ and fewer post-translational modifications as compared to that of the cells transfected with MIZ-1 (see Discussion).

\section{Discussion}

To date, there has been no report to demonstrate that Hsp90 inhibition leads to down-regulation of MYC and MYCN. In this study, we have shown that Hsp90 inhibition rapidly destabilizes MYC and MYCN proteins in unfavorable neuroblastoma cells. Although the exact mechanism by which Hsp90 inhibition causes destabilization of MYC and MYCN is not clear, our results suggest that MYC and MYCN are among the Hsp90 client proteins. In addition, the AKT pathway is known to stabilize MYC and MYCN $(26,27)$. Since treatment of neuroblastoma cells with 17-DMAG results in downregulation of AKT, one could explain the destabilization of MYCN and MYC as a result of AKT inactivation.

Our data also suggest that there is yet an additional mechanism for MYCN and MYC destabilization in neuroblastoma cells with an intact p53 pathway. As described, inhibition of Hsp90 by 17-DMAG up-regulates p53 expression and concomitantly destabilizes MYCN (IMR5, CHP134) and

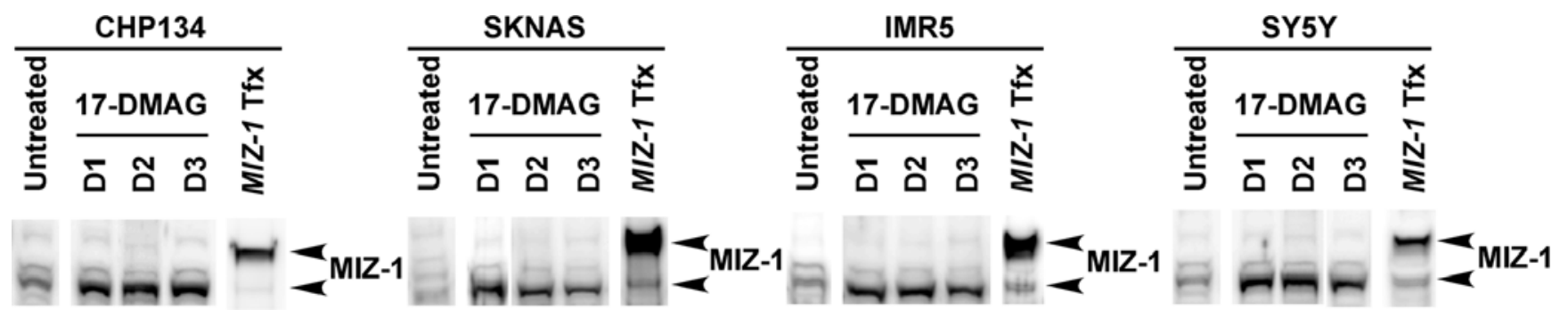

Figure 8. Treatment of neuroblastoma cell lines with 17-DMAG results in an induction of MIZ-1 protein expression. CHP134, SKNAS, IMR5 and SY5Y were treated with 17-DMAG $(5 \mu \mathrm{M})$ for 1, 2 and 3 days. The cells were harvested and subjected to Western blot analysis. MIZ-1-transfectants (designated as MIZ-1 Tfx) were included as positive controls (see Materials and methods). For the drug-treated samples, total protein (20 $\mu \mathrm{g})$ was loaded per lane. For the MIZ-1-Tfx samples, total protein $(5 \mu \mathrm{g})$ was loaded, with the exception of MIZ-1 IMR5 Tfx (2.5 $\mu \mathrm{g}$ protein was loaded). Anti-MIZ-1 rabbit polyclonal antibody (H-190, Santa Cruz Biotechnology) was used to detect MIZ-1 protein. Similar results were obtained when anti-MIZ-1 polyclonal antibodies (SC-5987 and SC-5984, Santa Cruz Biotechnology) were used (data not shown). 

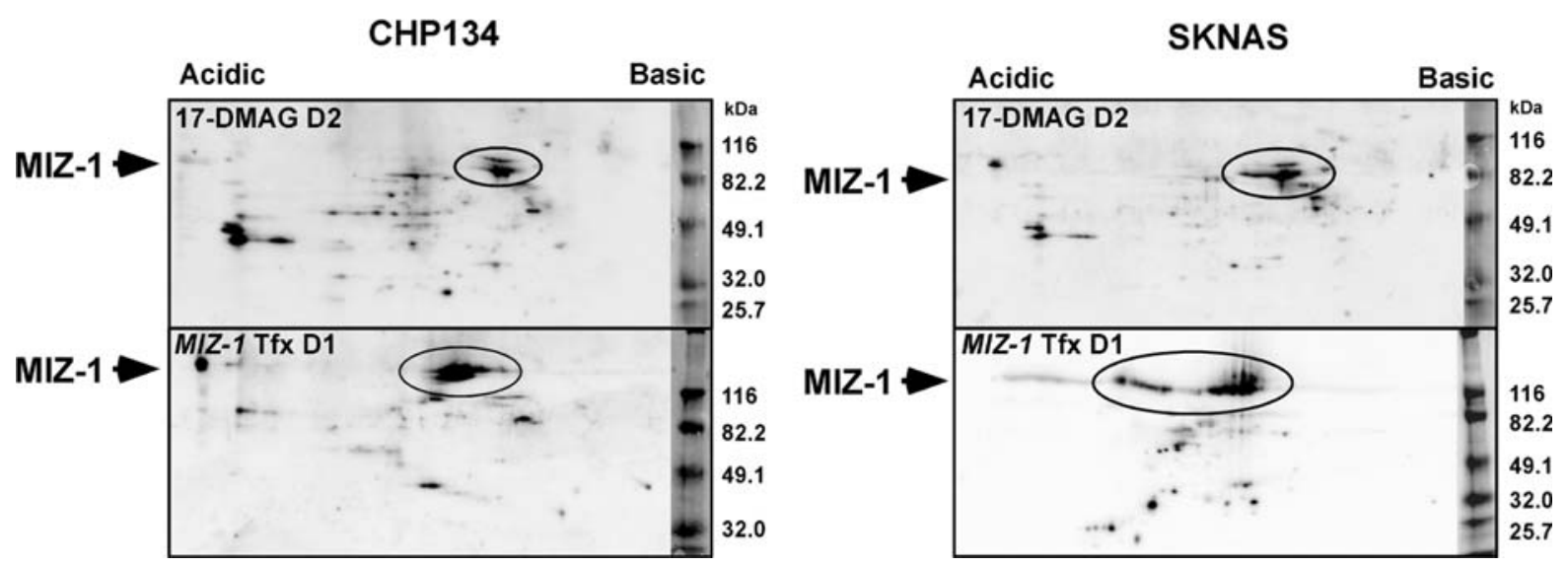

Figure 9. 2-D gel analysis confirms induction of MIZ-1 protein in the 17-DMAG-treated CHP134 and SKNAS. CHP134 and SKNAS were treated with 17-DMAG $(5 \mu \mathrm{M})$ for two days. The cells were harvested and subjected to 2-D gel analysis. For the transfectant controls, CHP134 and SKNAS were transfected with MIZ-1 (see Materials and methods) for $24 \mathrm{~h}$, and the MIZ-1-transfected cells (MIZ-1 Tfx) were subjected to 2-D gel analysis. Total protein (50 $\mu \mathrm{g}$ ) was loaded for each sample, with the exception of MIZ-1-transfected SKNAS (20 $\mu \mathrm{g}$ of total protein were loaded). Anti-MIZ-1 rabbit polyclonal antibody (H-190, Santa Cruz Biotechnology) was used to detect MIZ-1 protein.

MYC (SY5Y). There is an inverse correlation between p53 expression and MYCN or MYC expression in 17-DMAGtreated cell lines (Figs. 2 and 3). This observation is consistent with our previous study, which shows that an elevated p53 expression results in a decreased MYCN expression in $M Y C N$-amplified neuroblastoma cells (20). However, the identity of p53 targets that mediate the destabilization of MYCN and MYC in the neuroblastoma cells remains to be determined.

Based on the data shown in Figs. 3 and 4, the induction of $\mathrm{p} 21^{\mathrm{WAF} 1}$ is likely p53-dependent (SY5Y) and p53-independent (IMR5). It is not clear why CHP134 with the intact p53 pathway, fails to induce $\mathrm{p} 21^{\mathrm{WAF} 1}$ expression in response to p53 induction mediated by Hsp90 inhibition. However, based on our experience, it is more difficult to induce $\mathrm{p} 21^{\mathrm{WAF} 1}$ protein expression in CHP134 by drug treatments as compared to other cell lines $(20)$. Thus, the $\mathrm{p} 21^{\mathrm{WAF} 1}$ response mechanism to various environmental cues might be impaired in CHP134 cells.

Hsp90 is known to be key to the stability and function of many proteins that are important to growth and survival of cancer cells (14). To this end, our study has shown that Hsp90 inhibition also causes HDAC6 destabilization. It is known that HDAC6 is one of the tubulin deacetylases, and thus, HDAC6 depletion by Hsp90 inhibition results in hyperacetylation of tubulin (Fig. 6). As Hsp90 inhibition results in $\mathrm{G} 2 / \mathrm{M}$ arrest $(35,36)$, the hyper-acetylation of tubulin by Hsp90 inhibition may in part be involved in this phenomenon.

The depletion of AKT and other kinases by Hsp90 inhibition should have global consequences in the cell. It has been reported that MIZ-1 can be phosphorylated by AKT (37). The induction of MIZ-1 protein with a smaller molecular weight and fewer post-translational modifications (Figs. 8 and 9) therefore may be due to the depletion of AKT and/or other protein kinases that phosphorylate the MIZ-1 protein. In addition, our study shows that Hsp90 inhibition up-regulates the expression of favorable neuroblastoma genes (EFNB2, MIZ-1, NTRK1). We have previously shown that favorable neuroblastoma genes are epigenetically silenced in unfavorable neuroblastoma cells, but their expression can be enhanced by the treatment of small molecule epigenetic modifiers, including 5-aza-2'-deoxycitidine and 4-phenylbutyrate (13). As we have shown that HDAC6 is destabilized by Hsp90 inhibition, epigenetic silencers such as other HDACs and/or DNA methyltransferases may be among the Hsp90 client proteins. Destabilization of epigenetic silencers by Hsp90 inhibition may in turn activate many genes silenced in unfavorable neuroblastoma cells, including those described in this study.

In summary, our data suggest that Hsp90 inhibition suppresses the malignant phenotype of neuroblastoma through multiple pathways. Furthermore, activation of the p53 pathway and destabilization of MYC and MYCN are important mechanisms to the growth suppressive effect mediated by Hsp90 inhibition in neuroblastoma.

\section{Acknowledgements}

We would like to thank Autumn Fox for her critical reading, and Dr Naohiko Ikegaki for the MYCN antibody NCM II 100 and his critical reading of the manuscript. This work was supported by NIH grant CA97255 (X.X.T).

\section{References}

1. Maris JM, Hogarty MD, Bagatell R and Cohn SL: Neuroblastoma. Lancet 369: 2106-2120, 2007.

2. Modak S and Cheung NK: Neuroblastoma: Therapeutic strategies for a clinical enigma. Cancer Treat Rev 36: 307-317, 2010.

3. Brodeur GM, Seeger RC, Schwab M, Varmus HE and Bishop JM: Amplification of $\mathrm{N}$-myc in untreated human neuroblastomas correlates with advanced disease stage. Science 224: 1121-1124, 1984.

4. Schwab M, Varmus HE, Bishop JM, et al: Chromosome localization in normal human cells and neuroblastomas of a gene related to c-myc. Nature 308: 288-291, 1984.

5. Seeger RC, Brodeur GM, Sather H, et al: Association of multiple copies of the $\mathrm{N}$-myc oncogene with rapid progression of neuroblastomas. New Engl J Med 313: 1111-1116, 1985.

6. Fredlund E, Ringner M, Maris JM and Pahlman S: High Myc pathway activity and low stage of neuronal differentiation associate with poor outcome in neuroblastoma. Proc Natl Acad Sci USA 105: 14094-14099, 2008. 
7. Tang XX, Zhao H, Robinson ME, et al: Implications of EPHB6, EFNB2, and EFNB3 expressions in human neuroblastoma. Proc Natl Acad Sci USA 97: 10936-10941, 2000.

8. Combaret V, Gross N, Lasset C, et al: Clinical relevance of CD44 cell-surface expression and N-myc gene amplification in a multicentric analysis of 121 pediatric neuroblastomas. J Clin Oncol 14: 25-34, 1996.

9. Ikegaki N, Gotoh T, Kung B, et al: De novo identification of MIZ-1 (ZBTB17) encoding a MYC-interacting zinc-finger protein as a new favorable neuroblastoma gene. Clin Cancer Res 13: 6001-6009, 2007.

10. Kogner P, Barbany G, Dominici C, Castello MA, Raschella G and Persson H: Coexpression of messenger RNA for TRK protooncogene and low affinity nerve growth factor receptor in neuroblastoma with favorable prognosis. Cancer Res 53: 2044-2050, 1993.

11. Nakagawara A, Arima-Nakagawara M, Scavarda NJ, Azar CG Cantor AB and Brodeur GM: Association between high levels of expression of the TRK gene and favorable outcome in human neuroblastoma. N Engl J Med 328: 847-854, 1993.

12. Suzuki T, Bogenmann E, Shimada H, Stram D and Seeger RC: Lack of high-affinity nerve growth factor receptors in aggressive neuroblastomas. J Nat Cancer Inst 85: 377-384, 1993.

13. Tang XX, Robinson ME, Riceberg JS, et al: Favorable neuroblastoma genes and molecular therapeutics of neuroblastoma. Clin Cancer Res 10: 5837-5844, 2004.

14. Banerji U: Heat shock protein 90 as a drug target: some like it hot. Clin Cancer Res 15: 9-14, 2009.

15. Bagatell R, Beliakoff J, David CL, Marron MT and Whitesell L: Hsp90 inhibitors deplete key anti-apoptotic proteins in pediatric solid tumor cells and demonstrate synergistic anticancer activity with cisplatin. Int J Cancer 113: 179-188, 2005.

16. Kang J, Kamal A, Burrows FJ, Evers BM and Chung DH: Inhibition of neuroblastoma xenograft growth by Hsp90 inhibitors. Anticancer Res 26: 1903-1908, 2006.

17. Smith MA, Morton CL, Phelps DA, et al: Stage 1 testing and pharmacodynamic evaluation of the HSP90 inhibitor alvespimycin (17-DMAG, KOS-1022) by the pediatric preclinical testing program. Pediatr Blood Cancer 51: 34-41, 2008

18. Ikegaki $\mathrm{N}$ and Kennett RH: Glutaraldehyde fixation of the primary antibody-antigen complex on nitrocellulose paper increases the overall sensitivity of immunoblot assay. J Immunol Methods 124: 205-210, 1989

19. Tang XX, Evans AE, Zhao H, et al: High-level expression of EPHB6, EFNB2, and EFNB3 is associated with low tumor stage and high TrkA expression in human neuroblastomas. Clin Cancer Res 5: 1491-1496, 1999.

20. Torres J, Regan PL, Edo R, et al: Biological effects of induced MYCN hyper-expression in MYCN-amplified neuroblastomas. Int J Oncol 37: 983-991, 2010.

21. Nakamura Y, Ozaki T, Niizuma H, Ohira M, Kamijo T and Nakagawara A: Functional characterization of a new p53 mutant generated by homozygous deletion in a neuroblastoma cell line. Biochem Biophys Res Commun 354: 892-898, 2007.

22. Goldschneider D, Horvilleur E, Plassa LF, et al: Expression of C-terminal deleted p53 isoforms in neuroblastoma. Nucleic Acids Res 34: 5603-5612, 2006.

23. Basso AD, Solit DB, Chiosis G, Giri B, Tsichlis P and Rosen N: Akt forms an intracellular complex with heat shock protein 90 (Hsp90) and Cdc37 and is destabilized by inhibitors of Hsp90 function. J Biol Chem 277: 39858-39866, 2002
24. Hostein I, Robertson D, DiStefano F, Workman P and Clarke PA: Inhibition of signal transduction by the Hsp90 inhibitor 17-allylamino-17-demethoxygeldanamycin results in cytostasis and apoptosis. Cancer Res 61: 4003-4009, 2001.

25. Sato S, Fujita N and Tsuruo T: Modulation of Akt kinase activity by binding to Hsp90. Proc Natl Acad Sci USA 97: 10832-10837, 2000.

26. Sears R, Nuckolls F, Haura E, Taya Y, Tamai K and Nevins JR: Multiple Ras-dependent phosphorylation pathways regulate Myc protein stability. Genes Dev 14: 2501-2514, 2000.

27. Chesler L, Schlieve C, Goldenberg DD, et al: Inhibition of phosphatidylinositol 3-kinase destabilizes Mycn protein and blocks malignant progression in neuroblastoma. Cancer Res 66: 8139-8146, 2006

28. Gross N, Balmas Bourloud K and Brognara CB: MYCN-related suppression of functional CD44 expression enhances tumorigenic properties of human neuroblastoma cells. Exp Cell Res 260: 396-403, 2000.

29. Eggert A, Grotzer MA, Ikegaki N, Liu XG, Evans AE and Brodeur GM: Expression of the neurotrophin receptor TrkA down-regulates expression and function of angiogenic stimulators in SH-SY5Y neuroblastoma cells. Cancer Res 62: 1802-1808, 2002

30. Chua YL, Ito Y, Pole JC, et al: The NRG1 gene is frequently silenced by methylation in breast cancers and is a strong candidate for the $8 \mathrm{p}$ tumour suppressor gene. Oncogene 28 : 4041-4052, 2009

31. Liu T, Laurell C, Selivanova G, Lundeberg J, Nilsson P and Wiman KG: Hypoxia induces p53-dependent transactivation and Fas/CD95-dependent apoptosis. Cell Death Differ 14: 411-421, 2007

32. Herold S, Wanzel M, Beuger V, et al: Negative regulation of the mammalian UV response by Myc through association with Miz-1. Mol Cell 10: 509-521, 2002.

33. Staller P, Peukert K, Kiermaier A, et al: Repression of p15INK4b expression by Myc through association with Miz-1. Nat Cell Biol 3: 392-399, 2001.

34. Wu S, Cetinkaya C, Munoz-Alonso MJ, et al: Myc represses differentiation-induced p21CIP1 expression via Miz-1-dependent interaction with the p21 core promoter. Oncogene 22: 351-360, 2003.

35. Niikura Y, Ohta S, Vandenbeldt KJ, Abdulle R, McEwen BF and Kitagawa K: 17-AAG, an Hsp90 inhibitor, causes kinetochore defects: a novel mechanism by which $17-\mathrm{AAG}$ inhibits cell proliferation. Oncogene 25: 4133-4146, 2006.

36. Zajac M, Moneo MV, Carnero A, Benitez J and MartinezDelgado B: Mitotic catastrophe cell death induced by heat shock protein 90 inhibitor in BRCA1-deficient breast cancer cell lines. Mol Cancer Ther 7: 2358-2366, 2008.

37. Wanzel M, Kleine-Kohlbrecher D, Herold S, et al: Akt and 14-3-3eta regulate Miz1 to control cell-cycle arrest after DNA damage. Nat Cell Biol 7: 30-41, 2005.

38. Ikegaki N, Bukovsky $\mathrm{J}$ and Kennett RH: Identification and characterization of the NMYC gene product in human neuroblastoma cells by monoclonal antibodies with defined specificities. Proc Natl Acad Sci USA 83: 5929-5933, 1986. 